\title{
Bridging Industry to Beamline through an Advanced Laboratory-Based Characterisation Facility
}

\author{
Richard E Johnston ${ }^{*}$, Cameron Pleydell-Pearce ${ }^{1}$, Alan Clarke², Kyriakos Mouzakitis ${ }^{2}$, Leon Wechie ${ }^{2}$, \\ Ling $\mathrm{Xu}^{3}$, Ric Allott ${ }^{3}$ \\ 1. Advanced Imaging of Materials (AIM) Facility, College of Engineering, Swansea University, \\ Swansea, UK. \\ 2. TWI Technology Centre (Wales), Port Talbot, UK. \\ 3. Science and Technology Facilities Council (STFC), Harwell, UK. \\ * Corresponding author: r.johnston@swansea.ac.uk
}

The Science and Technology Facilities Council (STFC) is a world leading multidisciplinary science and technology organisation. Its facilities provide a range of research techniques using neutrons, muons, lasers and x-rays, and high performance computing and complex analysis of large data sets. In Wales, a regional pilot beamline-bridging centre, a partnership between an academic institution (Swansea University) and a research and technology organization (RTO, TWI Wales) has been setup to develop new relationships with industry partners in the region, leading to new engagements with STFC facilities (Fig.1). This will generate impacts for the company, people, and wider society in Wales. Impact could be new products, improved understanding of manufactured products, improved processing and materials development, or new research into medical and health interventions.

This partnership approach with industry in the region will increase the uptake of STFC facilities, in particular Diamond and ISIS, and the Central Laser Facility. The regional centre will attract a multidisciplinary research base from throughout Wales and increase their exposure to the UK's worldclass research facilities. The centre will be a hub for regional industry, generating impact through industrial research and development that was previously impossible or highly unlikely without this pilot bridging project.

There are challenges to engaging small-to-medium sized enterprises (SMEs) with large-scale scientific facilities such as those operated by STFC. The perceived gap between applied demand-led manufacturing, and seemingly high-level unattainable science presents a communication and translation challenge. In addition, SMEs have limited resource for research-based approaches to product development/improvement, coupled with constraints on staff time and capabilities to capture and analyse potentially large data. The regional centre, embedded locally, will bridge this perceived gap via a partnering approach, teaming the local company with beamline-industry-bridging officers (BIBOs) to identify the challenges, scope solutions, design experiments, carry out lab-based feasibility studies, and if beneficial, identify STFC facilities which could benefit the company. Once data is collected, this relationship will continue, with the BIBO's expertise and regional proximity to the company enabling the subsequent advanced analyses. This process will increase the likelihood of implementation of the findings in the company's activities, functioning as an impact pathway. Finally, the regional centre will help populate impact-tracking documentation in collaboration with the company to monitor and quantify the effects of the activity enabled by the regional pilot centre on both the company and the region.

So far the regional centre has identified common pathways to engaging local business, which include prior contact with industrial partners that either sponsor postgraduate projects with Swansea University, 
or who initiate contact through other business engagement touchpoints such as work-based learning initiatives. TWI can utilise its membership base within the region, along with identification of previous research projects requiring facilities beyond the current state-of-the-art. Also, a number of additional challenges have been identified through the operation, such as intellectual property (IP) considerations and data management. Through the life of this pilot project, the approach for engaging industry with central facilities is being evaluated through data collection and impact tracking, and will inform similar activities across different territories [2].

\section{References:}

[1] Welsh Government, Innovation Wales, (August 2015)

http:/gov.wales/docs/det/publications/140313innovationstrategyen.pdf

[2] The authors would like to acknowledge the financial support of the Bridging for Innovators Programme funded by BEIS, the Science and Technology Facilities Council (STFC) Grant No. ST/R006105/1, and the Engineering and Physical Sciences Research Council (EPSRC) Grant No. EP/M028267/1, and the European Social Fund (ESF) through the European Union's Convergence programme administered by the Welsh Government

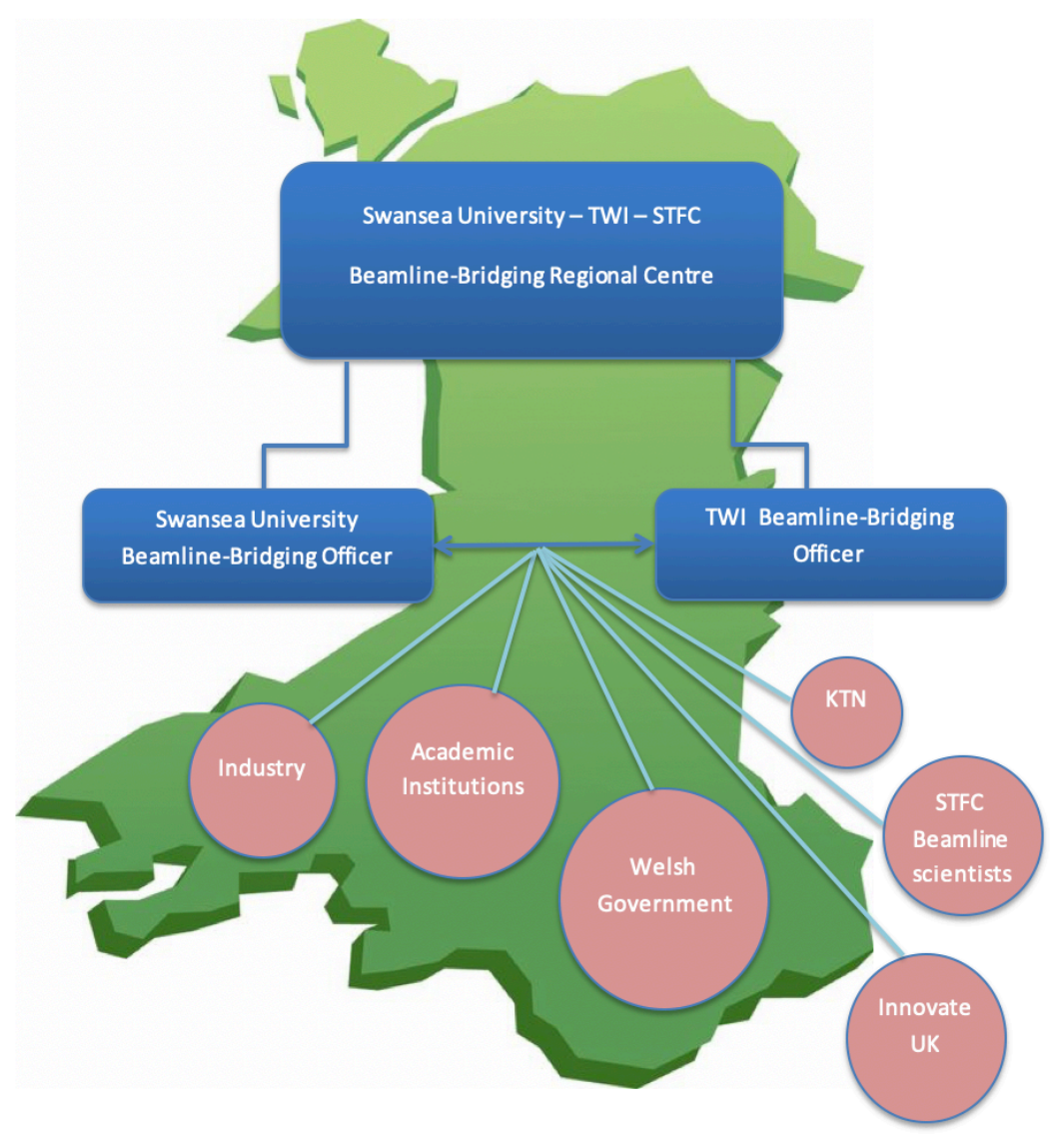

Figure 1. Wales Regional Industry-Beamline Bridging Centre schematic. Linking industry, academia, large-scale central facilities, government, industry liaison bodies. 